\title{
A GENERALIZATION OF THE AZ IDENTITY
}

\author{
RUDOLF AHLSWEDE and NING CAI
}

Received July 2, 1990

The identity discovered in [1] can be viewed as a sharpening of the LYM inequality ([3], [4], [5]). It was extended in [2] so that it covers also Bollobás' inequality [6]. Here we present a further generalization and demonstrate that it shares with its predecessors the usefullness for uniqueness proofs in extremal set theory.

\section{Introduction}

A few years ago Ahlswede and Zhang [1] found the following identity.

Theorem $A Z_{1}$. For every family $A \subset 2^{\Omega}$ of non-empty subsets of $\Omega=\{1,2, \ldots, n\}$

$$
\sum_{X \subset \Omega} \frac{W_{\mathcal{A}}(X)}{|X|\left(\mid \begin{array}{l}
n \\
|X|
\end{array}\right)}=1, \text { where } W_{\mathscr{A}}(X)=\left|\bigcap_{X \supset A \in \mathcal{A}} A\right| \text {. }
$$

We associate with every $\mathscr{E} \subset 2^{\Omega}$ the upset $\mathcal{U}(\mathscr{E})=\{U \subset \Omega: U \supset$ $E$ for some $E \in \mathscr{E}\}$ and the downset $\mathscr{D}(\mathscr{E})=\{D \subset \Omega: D \subset E$ for some $E \in$ E̊\}.

When $\mathcal{A}$ is an antichain in the poset $\left(2^{\Omega}, \supset\right)$, then the identity becomes

$$
\sum_{X \in \mathcal{A}} \frac{1}{\left(\begin{array}{l}
n \\
|X|
\end{array}\right)}+\sum_{X \in \mathcal{U}(\mathcal{A}) \backslash \mathcal{A}} \frac{W_{\mathcal{A}}(X)}{|X|\left({ }_{|X|}^{n}\right)}=1 .
$$

The LYM inequality is obtained by omission of the second summand, which by definition of $W_{\mathscr{A}}$ can also be written in the form $\sum_{X \notin D(A)} \frac{W_{\mathcal{A}}(X)}{\left.|X|{ }_{|X|}^{n}\right)}$. We call this the deficiency of the inequality.

More generally, in [2] the Bollobás inequality was lifted to an identity. 
Theorem $A Z_{2}$. For two families $\mathcal{A}=\left\{A_{1}, \ldots, A_{N}\right\}$ and $\mathscr{B}=\left\{B_{1}, \ldots, B_{N}\right\}$ of subsets of $\Omega$ with the properties

(a) $A_{i} \subset B_{i}$ for $i=1,2, \ldots, N$

(b) $A_{i} \not \subset B_{j}$ for $i \neq j$

$$
\sum_{i=1}^{N} \frac{1}{\left(\begin{array}{c}
n-\left|B_{i} \backslash A_{i}\right| \\
\left|A_{i}\right|
\end{array}\right)}+\sum_{X \notin \mathscr{D}(\mathscr{B})} \frac{W_{\mathscr{A}}(X)}{|X|\left(\begin{array}{c}
n \\
|X|
\end{array}\right)}=1
$$

In [1] it was explained that Theorem $A Z_{1}$ gives immediately, what LYM does not, namely the uniqueness part in Sperner's Theorem. In [2] the uniqueness of an optimal configuration of unrelated chains of subsets due to Griggs, Stahl and Trotter [7] was proved with the help of Theorem $A Z_{2}$.

Recently, Körner and Simonyi [10] observed the LYM-type inequality:

For $\mathscr{A}=\left\{A_{1}, \ldots, A_{N}\right\}, \mathscr{B}=\left\{B_{1}, \ldots, B_{N}\right\} \subset 2^{\Omega}$ with

$$
\begin{gathered}
A_{i} \cap B_{i}=\emptyset, A_{i} \not \subset A_{j} \cup B_{j}, B_{i} \not \subset A_{j} \cup B_{j} \text { for } i \neq j \\
\sum_{i=1}^{N}\left(\begin{array}{c}
n-\left|A_{i}\right| \\
\left|B_{i}\right|
\end{array}\right)^{-1}+\left(\begin{array}{c}
n-\left|B_{i}\right| \\
\left|A_{i}\right|
\end{array}\right)^{-1}-\left(\begin{array}{c}
n \\
\left|A_{i}\right|+\left|B_{i}\right|
\end{array}\right)^{-1} \leq 1
\end{gathered}
$$

and they asked (Problem 2) "Is this inequality ever tight?".

This rather modest question was a challenging test of the power of the identities in [1], [2] or, more precisely, of the procedure to produce new identities described in [1].

The outcome is an Ahlswede-Zhang type identity (Theorem 1) which goes considerably beyond Theorem $A Z_{2}$. From a special case of this identity we derive $a$ full characterization of the cases with equality (Theorem 2) even for a generalized version of $(3)$. In other words we characterize the cases with deficiency zero.

\section{The identity}

Theorem 1. Suppose that for a family $\mathscr{B}=\left\{B_{1}, \ldots, B_{N}\right\}$ of subsets of $\Omega$ and a family $\mathscr{A}^{*}=\left\{\mathcal{A}_{1}, \ldots, \mathcal{A}_{N}\right\}$ of subsets of $2^{\Omega}$, where $\mathcal{A}_{i}=\left\{A_{i}^{t}: t \in T_{i}\right\}$ for a finite index set $T_{i}$, we have the properties

(a) $A_{i}^{t} \subset B_{i}$ for $t \in T_{i}$ and $i=1,2, \ldots, N$

(b) $A_{i}^{t} \not \subset B_{j}$ for $t \in T_{i}$ and $i \neq j$.

Then with $\mathcal{A}=\bigcup_{i=1}^{N} \mathscr{A}_{i}$

(4) $\sum_{i=1}^{N} \sum_{k=1}^{\left|T_{i}\right|}(-1)^{k-1} \sum_{S \subset T_{i},|S|=k}\left(\begin{array}{c}n-\left|B_{i}-\bigcup_{t \in S} A_{i}^{t}\right| \\ \left|\bigcup_{t \in S} A_{i}^{t}\right|\end{array}\right)^{-1}+\sum_{X \notin \mathscr{D}(\mathscr{B})} \frac{W_{\mathscr{A}}(X)}{|X|\left(|X|{ }_{|X|}^{n}\right)}=1$.

The specialisation $\left|T_{i}\right|=1$ for $i=1, \ldots, N$ gives Theorem $A Z_{2}$. The proof goes again by counting chains. A key tool in [2] was 
Lemma 1. For two sets $A, B \subset \Omega$ with $A \subset B$ exactly $\frac{n !}{\left(\begin{array}{c}n-|B \backslash A| \\ |A|\end{array}\right)}$ maximal chains in $\left(2^{\Omega}, \subset\right)$ meet $\{X: A \subset X \subset B\}$.

1

Using the principle of inclusion-exclusion this generalizes to

Lemma 2. For $B \subset \Omega$ and $\mathscr{C} \subset 2^{\Omega}$ with $C \subset B$ for all $C \in \mathscr{C}$ exactly

$$
n ! \sum_{k=1}^{|\mathscr{C}|}(-1)^{k-1} \sum_{\mathscr{C}^{\prime} \subset \mathscr{C},\left|\mathscr{C}^{\prime}\right|=k}\left(\begin{array}{c}
n-\left|B \backslash \cup_{C \in \mathscr{C}^{\prime}} C\right| \\
\left|\cup_{C \in \mathscr{C}^{\prime}} C\right|
\end{array}\right)^{-1}
$$

maximal chains in $\left(2^{\Omega}, \subset\right)$ meet $\{X: C \subset X \subset B$ for some $C \in \mathscr{C}\}$.

Proof of Theorem 1. The number of maximal chains leaving $\mathcal{U}(\mathcal{A})$ at $U$ is

$$
(n-|U|) ! W_{\mathcal{A}}(U)(|U|-1) !
$$

Since the sets $X_{i}=\left\{X: A_{i}^{t} \subset X \subset B_{i}\right.$ for some $\left.t \in T_{i}\right\}(i==1,2, \ldots, N)$ are disjoint we have

$$
\sum_{i=1}^{N} \sum_{X \in X_{i}}(n-|X|) ! W_{\mathcal{A}}(X)(|X|-1) !+\sum_{X \in \mathcal{U}(\mathcal{A})-U X_{i}}(n-|X|) ! W_{\mathcal{A}}(X)(|X|-1) !=n !
$$

By the definition of $W_{A}$ the last summand can be written in the form

$$
\begin{gathered}
\sum_{X \notin \mathscr{D}(\mathscr{R})} \frac{W_{\mathcal{A}}(X) n !}{|X|\left(\begin{array}{l}
n \\
|X|
\end{array}\right)} \text { and by Lemma } 2 \\
\sum_{X \in \mathscr{X}_{i}}(n-|X|) ! W_{\mathcal{A}}(X)(|X|-1) !=n ! \sum_{k=1}^{\left|T_{i}\right|}(-1)^{k-1} \sum_{S \subset T_{i},|S|=k}\left(\begin{array}{c}
n-\left|B_{i} \backslash \cup A_{i}^{t}\right| \\
\left|\cup_{t \in S} A_{i}^{t}\right|
\end{array}\right)^{-1} \cdot
\end{gathered}
$$

\section{On zero deficiency}

We characterize here a case of zero deficiency, that is, the property

$$
\sum_{X \notin \mathscr{D}(\mathscr{B})} \frac{W_{d}(X)}{|X|\left(\begin{array}{c}
n \\
|X|
\end{array}\right)}=0
$$

Theorem 2. Under the assumptions of Theorem 1 and the additional conditions

(c) $A_{i}^{t} \cap A_{i}^{t^{\prime}}=\emptyset$ for all $i$ and $t, t^{\prime} \in T_{i}$ with $t \neq t^{\prime}$

(d) $\left|T_{i}\right| \geq 2$ and $N \geq 2$ 
we have, that the identity

$$
\sum_{i=1}^{N} \sum_{k=1}^{\left|T_{i}\right|}(-1)^{k-1} \sum_{S \subset T_{i},|S|=k}\left(\begin{array}{c}
n-\left|B_{i}-\cup_{t \in S} A_{i}^{t}\right| \\
\left|\cup_{t \in S} A_{i}^{t}\right|
\end{array}\right)^{-1}=1
$$

holds exactly if

(i) $\left|A_{i}^{t}\right|=1$ for all $t \in T_{i}$ and all $i$.

(ii) $B_{1} \backslash \cup_{t \in T_{1}} A_{1}^{t}=B_{2} \backslash \cup_{t \in T_{2}} A_{2}^{t}=\ldots=B_{N} \backslash \cup_{t \in T_{N}} A_{N}^{t}=B$, say.

(iii) $\sum_{i=1}^{N}\left|T_{i}\right|=n-|B|$.

In words, the $B_{i}$ have a common part $B$ and each $B_{i}$ has a rest of singletons $A_{i}^{t}$. The $B_{i}$ 's exhaust $\Omega$.

In the proof we use a well-known identity, which follows by iterative application of Pascal's. identity.

Lemma 3. $\sum_{k=1}^{m}(-1)^{k-1}\left(\begin{array}{c}M \\ m-k\end{array}\right)=\left(\begin{array}{c}M-1 \\ m-1\end{array}\right)$.

Proof of Theorem 2. From (i) and (ii) we derive in terms of $\beta=|B|$

$$
\begin{aligned}
& \sum_{i=1}^{N} \sum_{k=1}^{\left|T_{i}\right|}(-1)^{k-1} \sum_{S \subset T_{i},|S|=k}\left(\begin{array}{c}
n-\left|B_{i} \backslash \cup_{t \in S} A_{i}^{t}\right| \\
\left|\cup_{t \in S} A_{i}^{t}\right|
\end{array}\right)^{-1} \\
& =\sum_{i=1}^{N} \sum_{k=1}^{\left|T_{i}\right|}(-1)^{k-1}\left(\begin{array}{c}
\left|T_{i}\right| \\
k
\end{array}\right)\left(\begin{array}{c}
n-\left(\beta+\left|T_{i}\right|\right)+k \\
k
\end{array}\right)^{-1} \\
& =\sum_{i=1}^{N} \sum_{k=1}^{\left|T_{i}\right|}(-1)^{k-1} \frac{\left|T_{i}\right| !\left(n-\left(\beta+\left|T_{i}\right|\right)\right) !}{\left(\left|T_{i}\right|-k\right) !\left(n-\left(\beta+\left|T_{i}\right|\right)+k\right) !} \\
& =\sum_{i=1}^{N} \frac{\left|T_{i}\right| !\left(n-\left(\beta+\left|T_{i}\right|\right)\right) !}{(n-\beta) !} \sum_{k=1}^{\left|T_{i}\right|}(-1)^{k-1}\left(\begin{array}{c}
n-\beta \\
\left|T_{i}\right|-k
\end{array}\right) \\
& =\sum_{i=1}^{N} \frac{\left|T_{i}\right| !\left(n-\left(\beta+\left|T_{i}\right|\right)\right) !}{(n-\beta) !} \frac{(n-\beta-1) !}{\left(\left|T_{i}\right|-1\right) !\left(n-\left(\beta+\left|T_{i}\right|\right)\right) !}
\end{aligned}
$$

by Lemma 3 , and now by (iii)

$$
=\sum_{i=1}^{N} \frac{\left|T_{i}\right|}{n-\beta}=1 \text {. }
$$

We assume now that (6) holds and derive (i), (ii), and (iii). By Theorem 1 we have deficiency zero, that is,

$$
W_{\mathcal{A}}(X)=0 \text { for all } X \notin \mathscr{D}(\mathscr{B}) \text {. }
$$


For the quantity

$$
m=\min \left\{\left|A_{i}^{t}\right|: 1 \leq i \leq N, t \in T_{i}\right\}
$$

we show first that it equals 1 , then we establish (i) and (ii), and finally (iii).

Step 1. W.l.o.g. we can assume $\left|A_{1}^{1}\right|=m$. For any $y \in \Omega \backslash B_{1}$ consider $A_{1}^{1} \cup\{y\}$. Thus clearly $A_{1}^{1} \cup\{y\} \not \subset B_{1}$ and by condition (b) also $A_{1}^{1} \cup\{y\} \not \subset B_{j}$ for $j \neq 1$. Therefore $A_{1}^{1} \cup\{y\} \notin \mathscr{D}(\mathscr{B})$ and by $(8) W_{\mathscr{A}}\left(A_{1}^{1} \cup\{y\}\right)=0$. By the minimality of $A_{1}^{1}$ in $\mathcal{A}$ and the definition of $W_{\mathcal{A}}$ every $m$-subset of $A_{1}^{1} \cup\{y\}$ must be in $\mathcal{A}$. In particular for any $a \in A_{1}^{1}$ the set $\left(A_{1}^{1} \backslash\{a\}\right) \cup\{y\}$ is in $\mathscr{A}$. Since it is not in $\mathscr{A}_{1}$ it must be in some $\mathcal{A}_{j}$ with $j \neq 1$.

W.l.o.g. we can assume it to be $A_{2}^{1}$. Furthermore, since $A_{1}^{1} \neq A_{1}^{2}$ we can require the $a$ choosen above to be from $A_{1}^{1} \backslash A_{1}^{2}$. Also, since by (b) $A_{1}^{2} \not \subset B_{2}$ there is $z \in$ $A_{1}^{2} \backslash B_{2}, z \neq a$. As previously we conclude that $A_{2}^{1} \cup\{z\} \notin \mathscr{D}(\mathscr{B})$ and that the $m$-set

$$
\left(A_{2}^{1} \cup\{z\}\right) \backslash\{y\}=\left(A_{1}^{1} \backslash\{a\}\right) \cup\{z\} \in \mathcal{A} .
$$

However, we also have $\left(A_{1}^{1}-\{a\}\right) \cup\{z\} \in \mathscr{A}_{1}$ and by $(c) A_{1}^{1} \cap\left(\left(A_{1}^{1}-\{a\}\right) \cup\{z\}\right)=\emptyset$. This implies $A_{1}^{1}=\{a\}$ and $m=1$.

Step 2. After relabelling we can assume now $A_{1}^{1}=\{1\}$ and $B_{1}=\{1,2, \ldots, \ell\}$. By the arguments in Step 1 we get $\{1, k\} \notin \mathscr{D}(\mathscr{B})$ and $\{1, k\} \supset\{k\} \in \mathscr{A}$ whenever $k>\ell$. By (b)for all $t \in T_{i}$ and $i \geq 2 A_{i}^{t}$ has an element, say $e$, with $e>\ell$. However, since $\{e\} \in A$ by (a), (b) and (c) actually $A_{i}^{t}$ must equal $\{e\}$. We thus know that $A_{i}^{t}$ is a singleton for all $i \geq 2$ and $t \in T_{i}$. Now we can let any $i \geq 2$ take the role of 1 in the previous argument and get that all $A_{1}^{t}$ are also singletons. We have proved (i).

Also we have arrived at the following configuration: $B_{i} \supset A_{i}=\cup_{t \in T_{i}} A_{i}^{t}$ and $B_{i} \cap A_{j}=\emptyset$ for $i \neq j$. We claim now that $B_{i}=A_{i} \cup C$, where $C=\Omega \backslash \cup_{i=1}^{N} A_{i}$. To see this, suppose that $c \in C$ and $c \notin B_{i}$. Then for any $a \in A_{i}\{a, c\} \notin \mathscr{D}(\mathscr{B})$ and thus $W_{\mathcal{A}}(\{a, c\})=0$. This, however, contradicts $W_{\mathcal{A}}(\{a, c\})=|\{a\}|=1$.

We have established (ii) with $B=C$. (6), together with the equations leading to (7), give now also (iii).

Finally we present a consequence of Theorem 2, which in particular gives a positive answer to the question of Körner and Simonyi mentioned in the Introduction.

Corollary. If we are given for $t=1,2$ and $i=1,2, \ldots, N$ sets $A_{i}^{t} \subset \Omega$ with $A_{i}^{1} \cap A_{i}^{2}=$ $\emptyset$ and $A_{i}^{t} \not \subset A_{j}^{1} \cup A_{j}^{2}$ for $t=1,2$ and $i \neq j$ then

$$
\sum_{i=1}^{N}\left(\begin{array}{c}
n-\left|A_{i}^{1}\right| \\
\left|A_{i}^{1}\right|
\end{array}\right)^{-1}+\left(\begin{array}{c}
n-\left|A_{i}^{2}\right| \\
\left|A_{i}^{2}\right|
\end{array}\right)^{-1}-\left(\begin{array}{c}
n \\
\left|A_{i}^{1}\right|+\left|A_{i}^{2}\right|
\end{array}\right)^{-1}=1
$$

exactly if

(i') $\left|A_{i}^{t}\right|=1$ for $t=1,2$ and $i=1,2, \ldots, N$ 
(ii') $n$ is even and $N=\frac{n}{2}$.

There is a direct proof of this Corollary which is shorter than the one via Theorem 2.

Proof. With the choice $\mathscr{B}_{i}=\bigcup_{t \in T_{i}} A_{i}^{t}$ formula (6) takes the form

$$
\sum_{i=1}^{N} \sum_{k=1}^{\left|T_{i}\right|}(-1)^{k-1} \sum_{S \subset T_{i},|S|=k}\left(\begin{array}{c}
n-\sum_{t \notin S}\left|A_{i}^{t}\right| \\
\sum_{t \in S}\left|A_{i}^{t}\right|
\end{array}\right)^{-1}=1
$$

and if $T_{i}=\{1,2\}$ for all $i$ this becomes (10). (i) specializes to (i'), (ii) is true by definition of $B_{i}$ with $B=\emptyset$. (iii) specializes to $N \cdot 2=n$ and thus (ii').

\section{On general cloud antichains}

A family $\mathscr{A}^{*}=\left\{\mathscr{A}_{1}, \ldots, \mathcal{A}_{N}\right\}$ of subsets of $2^{\Omega}$ is a cloud-antichain, if

$$
A_{i} \not \subset A_{j} \text { for } A_{i} \in \mathscr{A}_{i}, A_{j} \in \mathscr{A}_{j} \text { with } i \neq j \text {. }
$$

They have been analyzed in [2] for $N=2$. In particular, in the case $\left|\mathcal{A}_{i}\right|=M$ for $i=1, \ldots, N$ we are interested in the maximal length $N(n, M)$ of these antichains.

Clearly, for $A=\bigcup_{i=1}^{N} \mathcal{A}_{i}$

$$
W_{\mathcal{A}}(X)=W_{\mathcal{A}_{i}}(X) \text { for } X \in \mathcal{A}_{i}
$$

and therefore by Theorem $A Z_{1}$

$$
\sum_{i=1}^{N} \sum_{X \in \mathcal{A}_{i}} \frac{W_{\mathcal{A}_{i}}(X)}{|X|\left(\mid{ }_{|X|}^{n}\right)}+\sum_{X \in \mathcal{U}(\mathcal{A}) \backslash \mathcal{A}} \frac{W_{\mathcal{A}}(X)}{|X|\left(\left|{ }^{n}\right|\right)}=1
$$

Notice that $n ! \sum_{X \in \mathscr{B}} \frac{W \mathscr{B}(X)}{|X|(|X|)}$ counts the number, say $\sigma(\mathscr{B})$, of saturated chains meeting a member of $\mathscr{B}$.

We can derive from (13) a bound on $N(n, M)$, if we have a bound or even exact result for the following seemingly basic quantity:

$$
s(M, n)=\min \left\{\sigma(\mathscr{B}): \mathscr{B} \subset 2^{\Omega},|\mathscr{B}|=M\right\} .
$$

\section{References}

[1] R. Ahlswede, and Z. Zhang: An identity in combinatorial extremal theory, $A d$ vances in Mathematics 80 (2) (1990), 137-151.

[2] R. Ahlswede, and Z. ZHANG: On cloud-antichains and related configurations, Discrete Mathematics 85 (1990), 225-245. 
[3] K. Yамамото: Logarithmic order of free distributive lattices, J. Math. Soc. Japan 6 (1954), 343-353.

[4] L.D. MeshalKin: A generalization of Sperner's theorem on the number of subsets of a finite set, Theor. Probability Appl. 8 (1963), 203-204.

[5] D. Lubell: A short proof of Sperner's theorem, J. Combinatorial Theory 1 (1966), 299.

[6] B. Bollobás: On generalized graphs, Acta Math. Acad. Sci. Hungar. 16 (1965), 447-452.

[7] J.R. Griggs, J. Stahl, and W.T. Trotter: A Sperner theorem on unrelated chains of subsets, J. Comb. Theory, Ser. A 36 (1984), 124-127.

[8] K. Engel, and H.D.O.F. Gronau: Sperner Theory in Partially Ordered Sets, Texte zur Mathematik Bd. 78, Teubner, Leipzig, 1985.

[9] E. SPERNER: Ein Satz über Untermengen einer endlichen Menge, Math. Z. 27 (1928), 544-548.

[10] J. Körner, and G. Simonyi: A Sperner-type theorem and qualitative independence, J. Comb. Theory, Ser. A $\mathbf{5 9}$ (1992), 90-103.

Rudolf Ahlswede, Ning Cai

Universität Bielefeld,

Fakultät für Mathematik,

D-4800 Bielefeld 1, Germany

hollmann@math. 10.mathematic.

uni-bielefeld.de 